\title{
Stance phase mechanical characterization of transtibial prostheses distal to the socket: A review
}

\author{
Matthew J. Major, PhD; ${ }^{1-2 *}$ Laurence P. J. Kenney, PhD; ${ }^{2}$ Martin Twiste, PhD; ${ }^{2-3}$ David Howard, PhD $^{\mathbf{2}}$ \\ ${ }^{1}$ Northwestern University Prosthetics-Orthotics Center, Northwestern University Feinberg School of Medicine, Chicago, \\ $I L ;{ }^{2}$ Centre for Health Sciences Research, University of Salford, Greater Manchester, UK; ${ }^{3}$ UNIPOD, United National \\ Institute for Prosthetics \& Orthotics Development, University of Salford, Greater Manchester, UK
}

\begin{abstract}
Achieving the required functionality of a transtibial prosthesis during the stance phase of gait (e.g., shock absorption, close to normal roll-over characteristics, and smooth transition into swing) depends on the Amputee Independent Prosthesis Properties (AIPPs), defined here as the mechanical properties of the prosthesis that directly influence the performance of the amputee. Accordingly, if research studies are to advance the design of prostheses to achieve improved user performance, AIPPs must be a primary consideration. However, the majority of reported studies can be categorized as either human performance testing of commercial prosthetic components or AIPP characterization; only in a few notable cases have studies combined these two approaches. Moreover, very little consistency exists in the current methods used for AIPP characterization, thus making comparisons between the results of such studies very difficult. This article introduces a framework for studying prosthesis design, which includes AIPP characterization, human performance and/or gait simulation studies, and detailed design. This framework provides a structure for reviewing previous approaches to AIPP characterization, discussing both their merits and shortcomings and their use in previous experimental and simulation studies. For the purposes of this review, stance phase AIPP models have been categorized as either lumped parameter or roll-over shape based.
\end{abstract}

Key words: amputee, characterization, design, gait, lumped parameter, mechanical properties, prosthesis, roll-over, simulation, transtibial.

\section{INTRODUCTION}

The required functionality of a transtibial prosthesis during the stance phase of gait can be summarized as (1) providing early stance shock absorption during load acceptance, (2) adapting to the ground surface by plantarflexing to a stable foot flat position, (3) providing close to normal shank kinematics during stance by replicating the loaded roll-over shape of the normal foot, and (4) contributing to push-off and a close to normal transition into swing phase. Achieving this functionality depends on the Amputee Independent Prosthesis Properties (AIPPs). In this context, AIPPs are defined to be the mechanical properties of the prosthesis that directly influence the comfort and performance of the amputee (i.e., the properties that the amputee experiences) and that are not based on in vivo measurements that may include confounding influences

\footnotetext{
Abbreviations: AIPP $=$ Amputee Independent Prosthesis Property, ESAR = energy storage and return, $\mathrm{MeSH}=$ Medical Subject Headings, SACH $=$ solid ankle cushion heel.

*Address all correspondence to Matthew J. Major, PhD; Northwestern University Prosthetics-Orthotics Center, Northwestern University, 680 N Lake Shore Dr, Suite 1100, Chicago, IL 60611; 312-503-5731.

Email: Matthew-Major@Northwestern.edu

http://dx.doi.org/10.1682/JRRD.2011.06.0108
} 
related to compensatory gait biomechanics. AIPPs describe the properties of the prosthesis as a whole and include the roll-over shape, alignment of the roll-over shape, elastic properties, damping properties, and mass properties, which all directly affect amputee gait [1-4]. Conversely, the underlying design details (e.g., subcomponent geometries, material densities, and Young's moduli) are not considered to be AIPPs; rather, they determine the AIPPs and therefore only indirectly affect amputee gait.

To make this distinction clear, we consider the example of elastic properties. If two prosthetic feet have different AIPPs (e.g., elastic properties), then an amputee will be able to feel the difference. Note that, in this context, the elastic properties are those for the prosthesis as a whole (i.e., the combined effect of all subcomponents). Conversely, if the subcomponent geometries and their Young's moduli (not AIPPs) differ but the combined elastic properties are the same, then the amputee will not feel the difference. The fact that apparently quite different designs could have very similar AIPPs [5-7] may help explain why previous clinical investigations have often been unable to draw clear conclusions regarding the relative advantages of different prosthetic componentry [8-10]. It is reasonable to assume that if research studies are to advance the design of prostheses to achieve improved user performance, AIPPs should be a primary consideration.

Unfortunately, to date the vast majority of studies investigating the effects of prosthesis design on amputee performance have compared different products in terms of their biomechanical and physiological effects, without characterizing AIPPs [8-12]. Because the primary information distinguishing the prostheses is their trade names and/or classification (e.g., conventional or energy storage and return [ESAR] in the case of prosthetic feet [1]), this approach can only indicate their relative performance but cannot provide information on why a particular prosthesis performs better than the next. A smaller number of studies have characterized prostheses in terms of their AIPPs; however, with notable exceptions [7,13-22], very few authors have combined both types of study in an attempt to understand the correlations between AIPPs and their effects on amputee gait (comfort, biomechanics, and physiological performance). Furthermore, these correlations can also be investigated through simulations of amputee gait that incorporate AIPP-based prosthesis models. Only in this way will more generic information, as opposed to product-specific claims, become more widely available to inform future designs, prescription, and alignment procedures.

Because manufacturers do not disclose their in-house design approaches, it is not possible to state with certainty whether or not current design practice involves the explicit use of AIPPs. Nevertheless, the very limited focus on AIPPs in the literature suggests that many of the current studies of the effects of prosthetic components on gait do not further our understanding of the relationships between the mechanical properties of prostheses and amputee gait. This limited focus is demonstrated by the fact that of the 37 studies identified as suitable for potential inclusion in the systematic Cochrane review [10] on prescription of prosthetic ankle-foot mechanisms, only 3 described the AIPPs of the components used during testing. Given a better understanding of AIPPs and their influence on amputee gait, an effective approach to prosthesis design can be envisaged in which the first stage is to identify the required AIPPs for different amputee groups, either from published empirical data or by simulating amputee gait using an AIPP-based prosthesis model. Then, alternative design solutions (i.e., materials, geometry, and physical construction) can be assessed using standard engineering analysis techniques, such as finite element analysis, to establish whether the design solutions realize the required AIPPs.

This envisaged approach to design is outlined in Figure 1; the elements above the dashed line (Stage 1) represent the research that informs design. This research precedes design and uses in vivo gait analysis and/or gait simulation, together with AIPP measurement and/or simulation, to identify the desired AIPPs that we want new designs to achieve. In this context, in vivo testing measures amputee gait performance, whereas amputee independent testing measures AIPPs. An important feature of this approach is that the desired AIPPs are dependent on the amputee cohorts that are being tested or simulated. Furthermore, these cohorts should be grouped according to their motor capacities. Consequently, it may be possible to arrive at very different sets of desired AIPPs, and hence design criterion, for different amputee groups, for example, young, active amputees and older, less mobile amputees.

However, one factor that greatly limits the potential for applying the approach described in Figure $\mathbf{1}$ is the lack of an established and well-accepted framework for objectively and quantitatively describing AIPPs. Indeed, as discussed previously, few studies involve the measurement of AIPPs, and among those that do, there is very little consistency in the methods adopted. 


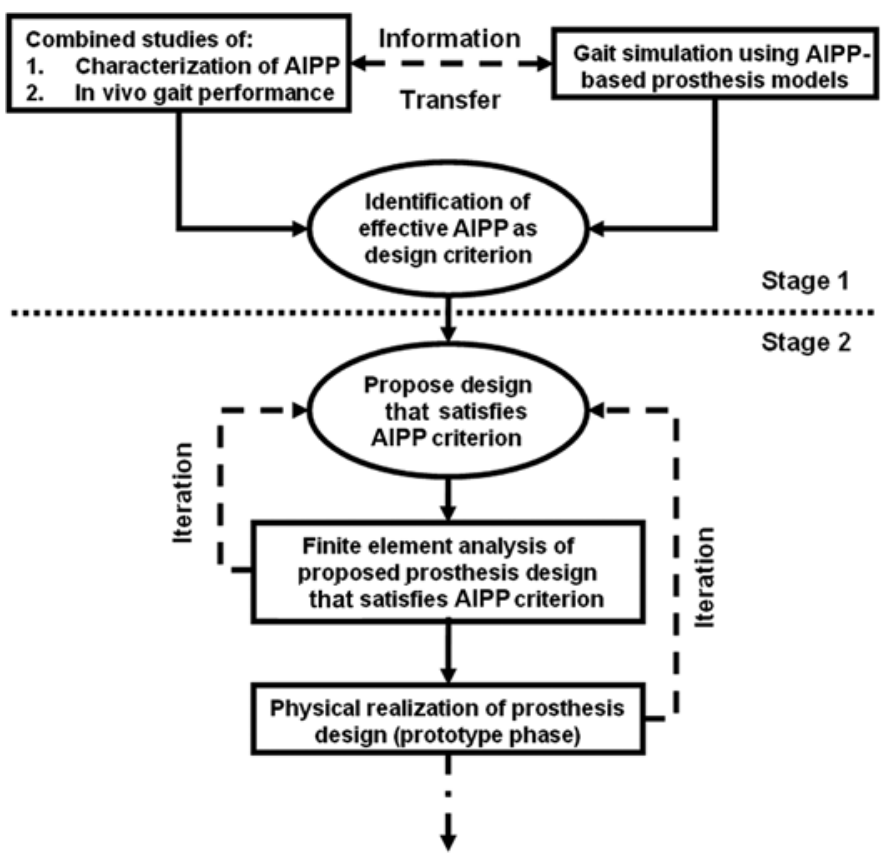

Figure 1.

Amputee Independent Prosthesis Property (AIPP)-based prosthesis design: Stage 1 = AIPP research studies. Stage $2=$ design and prototyping.

Therefore, the primary purpose of this article is to review alternative stance phase AIPP models, the methods previously used for measuring AIPPs, and how such models have been used in in vivo and in silico (numerical simulation) research studies. The scope of this review is limited to the passive properties of transtibial prostheses and does not include components capable of internal power generation. Because the focus of this article is stance phase AIPPs, mass properties are not discussed as their influence is predominantly in the swing phase [4].

\section{METHODS}

A primary search for relevant literature from 1970 to July 2011 was performed electronically using the MEDLINE and EMBASE databases with the following key words (Medical Subject Headings [MeSH] terms and free text words): "prosthesis," "prosthetic," "mechanical properties,” "leg,” “foot,” "pylon,” “shank,” “tibia,” "walking," "gait," "amputee," and "amputation." As an example, the resulting search in MEDLINE was as follows: ("Mechanical Phenomena" [MeSH]) AND ("Prostheses and Implants"
[MeSH] OR Prosthetic*) AND ("Leg” [MeSH] OR "Foot" [MeSH] OR Pylon* OR Shank* OR "Tibia” [MeSH]) AND ("Walking” [MeSH] OR "Gait” [MeSH] OR Amputee* OR "Amputation” [MeSH]). Following initial identification and review, we performed a secondary search using the references of articles identified in the primary search. This secondary search identified articles from relevant nonMEDLINE/EMBASE-indexed journals, such as the Journal of Prosthetics and Orthotics.

We read the abstracts of publications in which the title appeared to discuss a relevant topic and subsequently read the articles if relevance was confirmed by the information presented in the abstract. Articles were selected for inclusion in this review if the article reported the characterization of mechanical properties of any modular prosthetic components distal to the socket, or external fixation in the case of osseointegration, whether reported on its own or within the context of its effects on gait. Articles were excluded from this review if the article-

- Only reported fatigue testing of the components.

- Tested prostheses in which the socket and shank were constructed of a single material (e.g., monolimb prosthesis [23]; the scope of this review dealt specifically with components distal to the socket).

- Tested prostheses capable of internal power-generation,

- Tested components involved in studies on transfemoral amputee gait.

- Was written in a language other than English.

\section{RESULTS}

The primary search resulted in 443 articles identified within MEDLINE and 410 identified within EMBASE. Following selection for relevance and including those resulting from the secondary search, we identified 36 articles that satisfied the criteria [2-3,5-7,13-22,24-44]. In addition, five relevant review articles were identified [1,89,11-12]. In the following section, the approaches to characterization are considered under two broad headings: lumped parameter based methods and roll-over curve based methods. First, the models themselves are reviewed, and then their use in combination with in vivo gait analysis or as inputs to numerical simulation is discussed. 


\section{DISCUSSION}

\section{Lumped Parameter Models}

Of the articles identified, 67 percent adopted the lumped parameter approach for the characterization of prosthetic feet. Such models use discrete mass, spring, and damper elements to represent the mechanical response of more complex, continuous structures to static and/or dynamic loading. Referring to Figure 2, the spring elements model the stiffness (reciprocal of compliance) of the prosthesis. The damper elements model energy dissipation within the prosthesis as a result of friction of various kinds (but approximated as being viscous). The advantage of lumped parameter models is their simplicity, with only a small number of parameters needing identification. Their disadvantage is that a single lumped parameter model represents the viscoelastic properties at only one location on the prosthesis and in only one direction; multiple models must be used to represent the properties at different moments during stance. The locations and directions, relative to the foot, at which the viscoelastic properties are measured are usually chosen to be representative of one or

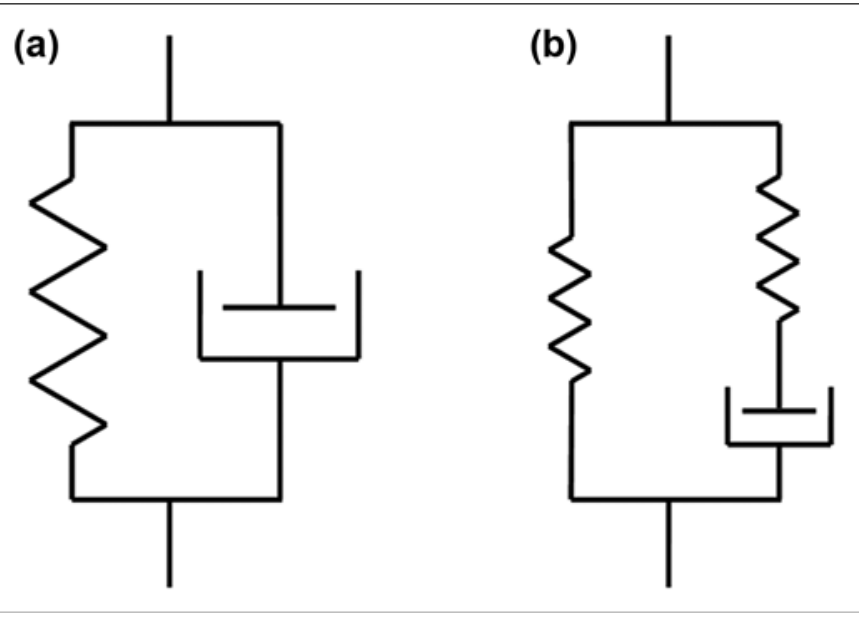

Figure 2.

Schematic illustration of (a) Voigt and (b) Kelvin lumped parameter models. For adapted Voigt model used in study by Klute et al. [29], equation for foot-ground reaction force, $F_{g}$, as function of displacement, $x$, was $F_{g}=a x^{b}+\operatorname{sign}(\dot{x}) c x^{d}|\dot{x}|^{e}$; where $a$ is stiffness coefficient, $c$ is damping coefficient, and $\operatorname{sign}(\dot{x})$ is 1 for $\dot{x} \geq 0$ and -1 for $\dot{X}<0$. Note inclusion of position-dependent factor in damping element. By setting exponents $b$ and $e$ to 1 and $d$ to 0 , this model would represent linear spring and damper, as used in Voigt model by Miller and Childress [15]. more key points in the gait cycle, such as heel-strike and push-off, but this is rather arbitrary and does not fully represent prosthesis behavior throughout stance. Lumped parameter models include the Maxwell (spring and damper in series), Voigt (spring and damper in parallel), and Kelvin or Standard Linear Solid (Maxwell model in parallel with a spring) [45]. The Maxwell model, however, is not suitable for modeling prosthetic feet as it predicts continuous creep under constant stress and eventual complete stress relaxation given constant strain. It is, thus, more suitable for modeling the behavior of fluids or softer materials, such as polymers [45].

Of the studies that only considered the mechanical characterization of components, a number of different justifications are cited by authors in support of the use of lumped parameter prosthesis models. Two studies reported that the motivation was to develop a standardized, accurate method of characterizing the mechanical properties of prosthetic feet, thus providing amputee independent comparative data [25-26]. Six further studies explicitly recognized that such an approach not only provides comparative data (in some cases with/without footwear), but also provides the potential to better understand how such properties relate to clinical benefits [5-6,27-29,42]. Although such studies provide potentially valuable information for comparison purposes, they lacked in vivo or gait simulation data to properly interpret their results. For the purposes of improving future designs, the AIPPs of the prostheses used during in vivo testing must be known with reasonable accuracy to understand the effects of different AIPPs on amputee gait. This can be achieved either through combined studies in which the actual prostheses used during in vivo gait analysis are tested to characterize their AIPPs or by referring to separate AIPP characterization studies in which nominally identical prosthetic components have been tested. The latter is only appropriate if there is confidence that good repeatability exists between nominally identical components. Despite the challenges of doing so, a small number of studies have undertaken combined studies using lumped parameter AIPP models, and these are discussed later. In a recent and highly relevant study, the AIPPs of a commercially available prosthetic foot were measured in order to replicate its stiffness properties in an experimental foot fabricated through selective laser sintering rapid prototyping [44]. The authors suggest using such a production method to provide a means for conducting systematic empirical studies of the effects of 
AIPPs on gait performance as required for Stage 1 of the process described in Figure 1.

Despite the frequent reporting of lumped parameter models, there is no consensus in the literature on which particular model is best suited for the characterization of prosthetic feet in terms of model complexity, accurate representation of properties, or clinical relevance. Previous studies have, for example, used the Voigt (e.g., Berge et al. [27] and Klute et al. [29]) or Kelvin model (e.g., Geil [25]) (Figure 2(a) and (b)). Many studies do not specify a particular lumped parameter model and simply report the stiffness and/or damping properties based on observing the response to static and/or time-varying loads [5-6,13$14,17,19-20,26,42,44]$. In this case, it is reasonable to assume that the Voigt model has been implicitly adopted as this is by far the most common engineering representation of viscoelastic resistance. In general, the justification for the choice of a particular approach has often not been made clear, although the fit of the model to the observed data is, unsurprisingly, sometimes cited.

Most studies focus on one particular aspect of the foot's response, such as the response to impact loads at heel-strike and, hence, model the response at just one or two points on the plantar surface of the foot. However, in one study, the foot was characterized by multiple, onedegree-of-freedom spring and damper models at 66 points along the foot [5]. This approach provides a representation of the foot's mechanical behavior throughout the entirety of stance (i.e., heel-strike to toe-off). Apart from two studies identified by the authors of this review [26,42], it is worth noting that all previous studies only consider the response to loading in the sagittal plane.

\section{Experimental Methods for Measuring AIPPs for Lumped Parameter Models}

This section discusses the process of estimating model parameters (i.e., stiffness and/or damping coefficients). These are determined experimentally using observations of the deflection of the component(s) in response to increasing levels of static and/or quasi-static loading or the deflection and velocity of the component(s) in response to dynamic loading, such as a step unloading or cyclical loading/unloading [5-7,13-15,17,25-26,28-30,42,44]. During testing, the prosthetic foot is typically attached to a rigidly clamped pylon and oriented in such a way to allow loads to be applied to either the surface of the heel or the surface of the metatarsal head region (forefoot) [13-14,25,28-30,44] so that the direction of the applied loads approximately corresponds with the direction of the ground reaction force at the relevant point during gait. Deflections are then measured, typically in the direction in which the load is applied. Load magnitudes and velocities, or kinetic energy values (in the case of impact studies), are typically chosen to correspond to values seen at heel-strike or push-off [56,13-14,25,29-30,44].

Additionally, stiffness and damping properties of vertical shock-absorbing pylons, represented as Voigt models, have been measured through the application of axial loads $[15,27]$. Furthermore, in addition to a study that reported a method of measuring the rotational movements of transverse rotation adaptors [35], one study measured the torque versus angular displacement behavior of these adaptors through the application of torsional loads under two constant rates of displacement $\left(0.5^{\circ} / \mathrm{s}\right.$ and $\left.60^{\circ} / \mathrm{s}\right)$ using a materials testing machine [31]. A couple of studies measured the linear stiffness of a modular, C-shaped articulating ankle joint alone, without a foot, through application of vertical loads (20 mm/min from $50 \mathrm{~N}$ to $1,230 \mathrm{~N}$ ) [19-20].

The study by Geil provides an example of a typical experimental setup in which a materials testing machine is used to subject a section of a prosthetic foot to controlled loading and unloading [25]. The foot is in contact with a low friction plate and load is applied perpendicular to that plate. Furthermore, the long axis of the foot is oriented at an angle of $12^{\circ}$ with respect to the plate to simulate the loading scenario during late stance (i.e., after heel lift). In this study, stiffness and damping coefficients of a Kelvin model (Figure 2(b)) were estimated through the combined results of a stress-relaxation test, creep test, and constant strain rate test (all three tests are necessary to produce three simultaneous equations and solve for the three model coefficients). The study by Miller and Childress provides another example of a custom test rig used to measure properties of a prosthetic foot and vertical shock-absorbing pylon [15]. The long axis of the prosthetic foot was oriented parallel to a near-frictionless plate (achieved using a ball bearing interface), and the "ball" of the prosthetic foot was loaded by a force perpendicular to the plate to simulate late stance and thereby estimate the stiffness coefficient of a Voigt model from the measured static force-displacement relationship. The vertical shock-absorbing pylon was loaded vertically, and the stiffness coefficient estimated by the same technique. In this setup, the damping coefficient was estimated from either the time response after a step unloading (pylon 
alone) or from the oscillation decay following a manually induced oscillation (pylon-foot assembly).

Interestingly, there appears to be little consistency between the techniques used to measure (nominally) the same properties. Not only does the particular type of lumped parameter model chosen vary between studies, but so also do the orientations of the foot relative to the loads, as well as the magnitudes and timing of the applied loads and the subsequent analysis of the results to derive model parameters.

For example, Saunders et al. [30], van Jaarsveld et al. [5], and Lehmann et al. [14] aimed to characterize the stiffness properties of the Otto Bock solid ankle cushion heel (SACH) foot, but each of these three studies used different approaches to loading and analysis. They all reported linear stiffness at the "heel strike" and "toe off" positions of an Otto Bock SACH foot, derived from the force-displacement plots when loading the foot within a materials testing machine. The study by Saunders et al. oriented the loading surface at $6^{\circ}$ and $178^{\circ}$ with respect to the long axis of the foot to simulate loading at heelstrike and toe-off, respectively [30]. In this setup, the applied load was perpendicular to the long axis of the foot, or at $96^{\circ}$ and $88^{\circ}$ with respect to the loading surface. In the study by van Jaarsveld et al., the long axis of the foot was oriented at $30^{\circ}$ and $145^{\circ}$ with respect to the loading surface to simulate heel-strike and toe-off, respectively [5]. In this setup, the direction of applied load was perpendicular to the loading surface and shear forces were eliminated by means of small displacements of the loading surface perpendicular to the direction of applied load. In the study by Lehmann et al., the point of load application was positioned at the "posterior extremity" of the heel and $14 \mathrm{~cm}$ anterior to the pylon attachment point for measurement at heel-strike and toe-off, respectively, and for both cases the load's line of action was "inclined" such that it intersected with the point at which the pylon would attach to the socket [14]. From the information provided, the long axis of the foot was oriented at $6^{\circ}$ and $164^{\circ}$ with respect to the loading surface to simulate heel-strike and toe-off, respectively, assuming that the direction of the applied load was perpendicular to the loading surface and the "posterior extremity" of the heel was located $5.5 \mathrm{~cm}$ posterior to the pylon attachment point (estimated from similar prosthetic feet, as not explicitly reported in the study).

Further, each study used different approaches to the calculation of stiffness coefficients. Saunders et al. calcu- lated the average slope of the force-displacement curve up to a displacement of $13.77 \mathrm{~mm}$ and $17.77 \mathrm{~mm}$ for heel-strike and toe-off, respectively [30]; van Jaarsveld et al. used curve fitting to smooth the data and calculated the slope at either the maximum force or displacement, defined as 1,000 $\mathrm{N}$ and $35 \mathrm{~mm}$ respectively, dependent on which occurred first during testing [5]. For the purpose of this review, we have calculated the average slope of the force-displacement curves presented by Lehmann et al. [14]. Perhaps unsurprisingly, the three studies reported very different results for toe-off and heel-strike stiffness: 28.7 and $44.0 \mathrm{~N} / \mathrm{mm}$, respectively, in the study by van Jaarsveld et al. [5]; 102.8 and $154.1 \mathrm{~N} / \mathrm{mm}$ in the study by Saunders et al. [30]; and 71.6 and $32.4 \mathrm{~N} / \mathrm{mm}$ in the study by Lehmann et al. [14]. Contrary to the results from the studies by Saunders et al. [30] and van Jaarsveld et al. [5], in the study by Lehmann et al. [14], the forefoot was estimated as having greater stiffness than the heel. This may be the result of differences in characterization techniques and/or differences in the particular model of Otto Bock SACH foot tested (which is not specified in any of the three studies).

Methods used to estimate damping properties also vary between studies. For instance, the studies by Lehmann et al. [13-14], Miller and Childress [15], and Sam et al. [32], estimated the damping properties by analyzing the oscillation of the prosthetic forefoot resulting from a step unloading. Other studies have estimated damping properties by measuring the hysteresis during controlled loading and unloading of a region of the prosthetic foot [5-6]. The studies that used the step unloading technique reported either the damped natural frequency of oscillation [13-14] or damping ratio [15,32], as calculated from the oscillation decay using the log-decrement method. The damping ratio and damped natural frequency can be used to calculate the damping coefficient for use in a lumped parameter model, but are dependent on both the stiffness of the prosthetic foot and the applied mass used to induce the oscillations used for their calculation. However, only the studies by Lehmann et al. [13-14] and Sam et al. [32] explicitly stated the applied masses used during testing, which were not the same.

The combined effects of the different approaches to modeling and the different approaches to estimating model coefficients make any sensible comparison of results between studies very difficult. 


\section{Combined In Vivo and AIPP Characterization Studies That Have Used Lumped Parameter Models}

As mentioned previously, many studies have used lumped parameter models simply as a means of comparing prosthetic feet in terms of their AIPPs but without indicating the effects of different AIPPs on amputee gait. Only a few studies have attempted to understand the relationships between AIPPs and amputee performance through in vivo gait analysis or gait simulation studies.

In the two studies by Lehmann et al., the linear stiffness properties of the heel and forefoot regions and the natural frequency of oscillation of the forefoot region of several different prosthetic feet were measured [13-14]. A gait analysis study of amputees walking on the different feet was then carried out, and the properties of the different feet were correlated with the gait analysis data. The authors reported that a greater range of prosthetic ankle angle during stance was associated with reduced forefoot stiffness [13-14], and increased maximum prosthetic side knee flexion moment during stance was associated with increased heel stiffness [14]. Furthermore, the authors observed that all of the tested prosthetic feet displayed damped natural frequencies of oscillation, with an applied mass of $68 \mathrm{~kg}$, which were considerably higher than the "stance phase" frequencies. The authors defined "stance phase frequency" as $1 / 2 T$, where $T$ was the average time from foot flat to toe-off as observed during gait analysis. They concluded that this mismatch between the natural frequency of the foot and the stance phase frequency may result in an untimely release of stored energy during the stance phase of amputee gait [13-14]. Additionally, correlations were drawn between subjective feedback on comfort and forefoot stiffness, with subjects showing a preference for prosthetic feet with a more compliant forefoot [13].

Miller and Childress used a lumped parameter model to characterize the mechanical properties of a prosthesis (the AIPPs) and compared the model coefficients with those of the anatomical limb, as reported in previously published literature [15]. In this study, Voigt models were used to represent a vertical shock-absorbing pylon and pylon-foot assembly. The authors noted that the overall stiffness coefficients for the pylon-foot assembly were remarkably insensitive to differences in the stiffness of the vertical shock-absorbing pylon. Further, the values of the model coefficients compared well to those of the physiological limb. The gait analysis part of the study simply observed differences in walking speed, vertical ground reaction force, and temporal parameters of gait with and without activation (i.e., enabling and disabling the telescoping function, respectively) of a vertical shockabsorbing pylon. Activation of the pylon, and hence increasing the vertical compliance of the prosthesis, was found to increase walking speed during fast walking (as well as decrease stance time of the prosthetic limb), increase vertical-ground reaction force of the prosthetic limb, and increase peak-to-peak vertical trunk motion for both fast walking and jogging. In this study, subjects preferred the prosthesis with the pylon activated. Apart from in vivo studies that compare differences in gait with and without the presence of vertical pylon compliance $[12,15,46-51]$, we are not aware of any studies of the effects on gait performance of changing the properties of vertical shock-absorbing pylons (AIPPs). However, a study by Coleman et al. made observations on the effects of pylon bending stiffness on transtibial amputee gait [36]. In this study, the authors reported characterizing the pylons as cantilever beams in order to calculate a stiffness value for each pylon. Results from this study indicated that use of the more flexible pylon produced anterior-posterior ground reaction forces that more closely matched the nonamputated limb.

Two recent combined AIPP and experimental studies investigated the effects of linear stiffness of a C-shaped articulating ankle component on gait. In combination with a standardized ESAR foot, the studies observed the effects of varying ankle joint stiffness (i.e., vertical stiffness) on lower-limb kinematics, kinetics, and muscle activity [19-20]. Each subject's gait was evaluated while walking along a level floor at approximately $1.2 \mathrm{~m} / \mathrm{s}$ under five conditions: no ankle joint component and two levels of ankle joint stiffness in two configurations ("C" opening facing anterior and posterior). Results indicated an inverse relationship between late stance peak dorsiflexion and peak vertical ground reaction force during unloading of the prosthetic limb. The authors also found that the more compliant anterior-facing ankle joint provided the greatest amount of late stance peak dorsiflexion [20]. Additionally, increased late stance dorsiflexion of the prosthetic limb provided by the more compliant ankle joint, irrespective of its configuration, also resulted in increased sound limb muscular activity for the purpose of body support [19].

Furthermore, apart from in vivo studies that compare differences in gait with and without the presence of rotational pylon compliance through the addition of a transverse 
rotation adaptor [12,52-54], we are not aware of any studies of the effects on gait performance of changing the properties of these adaptors (AIPPs). One case study was identified in which rotational stiffness (assumed to be those values provided by the manufacturer for the internal torsion component or "rod") was modulated in order to observe the effects on the specific kinematics of a golf swing (e.g., hip and shoulder rotation) of two transtibial amputees [37]. This study produced inconclusive results, which the authors believe is due to the small number of subjects and the intersubject differences (participants were one right- and one left-sided transtibial amputee).

The study by Postema et al. characterized the damping properties of several prosthetic feet by measuring the hysteresis seen in response to a loading profile representative of the stance phase of gait [7]. The prosthetic foot was subjected to a continuous purely vertical load while being rolled over a horizontal surface from simulated heel-strike (pylon angle of $32^{\circ}$ with respect to the horizontal) to toe-off (pylon angle of $40^{\circ}$ with respect to the horizontal). The mechanical work done by the loading device while the foot moved through this range of pylon angles was calculated as the integral of the applied vertical force with respect to vertical displacement. These results were used to compare the ESAR, and hence energy loss, as calculated from total ankle power during gait with that measured independent of the amputee in the test device. Results indicated that the energy storage measured with the test device was two to three times smaller than that calculated from total ankle power during gait, which the authors believe is primarily due to the differences in the method of calculating work done (i.e., integration of prosthetic ankle joint moment with respect to angular displacement from gait versus integration of applied vertical force with respect to vertical prosthesis deformation in the test device). This problem with the in vivo measurement techniques for calculation of ESAR in prosthetic feet has been investigated in studies by Prince et al. [55] and Geil et al. [56]. The study by Prince et al. presented an alternative method for calculating ESAR that accounted for both rotational and translational terms in the calculation of power [55]. Furthermore, the study by Geil et al. compared a conventional analysis (including only rotational terms) with the analysis that accounted for the translational terms also [56]. The authors reported that including the translational terms in their calculation of power indicated more effective ESAR (less energy dissipation) during the stance phase of gait when using an ESAR foot compared with that indicated by calculating energy using rotational terms alone [56].

The study by vd Water et al. [17] also characterized the damping properties (i.e., hysteresis) of an experimental prosthetic foot using the same technique and characterization rig as in the study by Postema et al. [7]. This foot was tested on three subjects, and its ESAR capabilities and effects on gait were compared to each subject's original prosthetic foot. The study found that the experimental prosthetic foot had a smaller hysteresis value (i.e., less energy dissipation) than any of the subjects' original prosthetic feet, which the authors believed would consistently decrease the energy cost of walking by returning more stored energy $[5,17]$. However, contrary to expectations, only two out of three subjects displayed a, sometimes minimal, reduction in metabolic rate (Joules/second) when walking with the experimental prosthesis as compared with their original prosthetic foot, perhaps because the energy was not returned at an appropriate time during gait.

It is worth noting that, in all of the studies comparing AIPPs with results from in vivo gait analysis presented here, the prosthesis was characterized off the body, after which standard clinical alignment procedures were used to setup the device on the amputee. It is known that alignment can significantly affect the mechanical behavior of prostheses [3,24], effectively changing their AIPPs and, hence, introducing a confounding effect.

One study observed the effects of prosthesis height and stiffness on the effective vertical stiffness of the legs (i.e., the ratio of peak vertical ground reaction force to whole body center of mass vertical displacement) during running for two unilateral transtibial amputees [43]. The prosthesis stiffness was varied independent of height by using the same model of prosthesis but with different stiffness levels. However, contrary to previous studies, the prosthesis stiffness for each condition was estimated as the ratio of peak vertical ground reaction force to peak prosthetic displacement along its longitudinal axis during gait (i.e., massless linear spring model). The effective vertical stiffness during weight-bearing on the prosthetic limb was not affected by changes in prosthesis height or stiffness, because of changes in other gait variables (e.g., peak vertical ground reaction force), suggesting perhaps a preference to maintain a consistent whole-body vertical stiffness [43]. 
Simulation Studies That Have Used Lumped Parameter AIPPs

Lumped parameter models have also been used in computer simulation studies to predict the effects of different prosthesis properties on amputee gait. In a study by Klute and Berge, the prosthetic limb of the transtibial amputee was modeled in order to simulate the influence of the prosthetic foot, shoes, amputee mass characteristics, and impact velocity on the vertical component of the ground reaction force at heel-strike [38]. Various prosthetic feet (with and without shoes) were characterized using the Voigt model as described previously [29]. The amputee was also represented using several Voigt models to simulate the upper rigid body (superior to the residual limb), the lower rigid body (shoe, prosthesis, and residual limb), and oscillating soft tissue masses (upper body soft tissue superior to the residual limb and residual limb soft tissue). Results from this model were validated with results from in vivo experimentation. A sensitivity analysis was then conducted using the lumped parameter simulation model by systematically adjusting the stiffness and damping coefficients of the prosthetic foot and shoe in order to observe their effect on the vertical ground reaction force. This simulation study [38], in combination with the experimental AIPP characterization study by the same authors [29], is an example of the type of study that could contribute to Stage 1 of the conceptual design approach described in Figure 1. Within the limitations acknowledged by the authors, this simulation can be used as a tool for identifying effective AIPPs with respect to the design objective of minimizing the vertical ground reaction force at heel-strike.

Another simulation study used a numerical musculoskeletal model to investigate the effects of an ESAR prosthetic foot-ankle mechanism on trunk support, forward propulsion, leg swing initiation, and muscle activation patterns required to produce a normal, symmetric gait pattern [39]. The prosthetic foot-ankle mechanism was modeled as a rotational viscoelastic ankle joint, the parameters of which were derived from data reported in the experimental study by Lehmann et al. [14]. This simulation identified how the ESAR prosthesis stored and returned energy during the stance phase of gait and how this compared with muscle contractions and associated work during nonamputee walking. Additionally, this study was able to identify muscle compensatory strategies employed by amputees to produce a symmetric gait pattern. This study demonstrates the usefulness of simulation in understanding amputee gait and its potential as a tool to systematically investigate the effects of different AIPPs on gait performance.

\section{Roll-Over Models}

A model that begins to bridge the gap between characterizing the mechanical properties of the prosthesis and its in vivo functional performance is the roll-over shape model $[2,40]$. The roll-over shape is a spatial mapping of the center of pressure location along the plantar surface of the foot relative to a shank-based coordinate frame. The roll-over shape can be measured in an amputeeindependent manner from data generated using a test rig [2-3,16,18,32-34], in which case it can be considered to be part of the AIPPs description of the prosthesis. By varying the applied loads, a family of roll-over shapes can be produced, which provide a representation of foot stiffness. A single roll-over shape can also be measured in vivo from continuous data produced during the stance phase of gait $[2,24]$. This in vivo roll-over shape is a function of the prosthetic foot's mechanical properties, its alignment, and the particular forces acting on the foot during the stance phase of gait. The stance phase forces are dependent on the amputee's physical characteristics and walking style; therefore, the in vivo roll-over shape is clearly not an AIPP.

Hansen et al. showed that prostheses with very different test rig (AIPP) roll-over shapes can be aligned to produce similar in vivo roll-over shapes, particularly in terms of the position and orientation of the roll-over curve, but not its curvature [24]. Hence, when establishing correlations between prosthesis properties and in vivo gait performance, it is the AIPP roll-over shape obtained from test rig data that should represent these properties. Having said this, because alignment is a function of the prosthetic components and their adjustment, it should also be part of the AIPPs description of the prosthesis. Therefore, in combined studies that aim to understand the relationships between AIPPs and amputee gait, alignment should be systematically controlled. Alignment by a prosthetist introduces unknown confounding effects as it is a result of a subjective process that, by definition, establishes different AIPPs for each subject. While other AIPPs, such as foot stiffness properties, can change the curvature of the roll-over curve, its position and orientation relative to the shank can be completely controlled by alignment. So we suggest that, in systematic AIPP studies, the alignment should be set to achieve a defined position and orientation of the roll-over curve with respect to the residual tibia. 
The roll-over shape model is a more intuitive representation of the mechanical properties of transtibial prostheses than the lumped parameter approaches discussed previously, combining the effects of foot geometry, alignment, and stiffness properties (via a family of curves for different loads). In previous studies, damping properties have been measured in addition to, but independent of, the roll-over shapes by application of a cyclical load to a section of the prosthetic foot (e.g., forefoot) [32,34], similar to the procedure described in the study of Miller and Childress [15]. However, the viscoelastic effects have yet to be integrated into the roll-over model. Additionally, the previously published versions of the roll-over shape model $[2,32-33,40]$ do not include measurements of shear stiffness (i.e., linear stiffness tangential to the support surface).

\section{Combined In Vivo and AIPP Characterization Studies That Have Used Roll-Over Models}

The AIPP roll-over model has also been used in combination with in vivo gait analysis to explore the relationships between AIPPs and gait. Hansen et al. investigated the relationship between roll-over shape arc length and gait kinematics and kinetics using the custom-designed "Shape\&Roll" prosthetic foot [16]. This foot was constructed of copolymer plastic in which the changes to its in vivo roll-over shape were achieved through cutouts made in the dorsal aspect of the foot. Therefore, the foot became highly stiff in each of its loaded configurations, and the changes in its behavior were assumed to be primarily due to changes in geometry, not the elastic properties of the foot. In this study, correlations were made between the rollover shape arc length and gait performance measures. For example, it was found that reducing the arc length resulted in reduced walking speed and prosthetic ankle dorsiflexion moment and an increased first peak of the vertical ground reaction force on the sound limb.

As a follow-up to the this study, two additional studies by Klodd et al. also used the "Shape\&Roll" prosthetic foot in order to study the effects of forefoot roll-over shape on gait kinematics, kinetics, and oxygen cost [2122]. Changes to forefoot roll-over shape were achieved in a foot of the same length by increasing the number of cutouts made in the dorsal aspect of the forefoot. Although the aim was to create five foot conditions with different forefoot stiffnesses, the cutouts were designed such that each foot condition would achieve a specific in vivo rollover shape radius, and hence, primarily geometries, not stiffness properties, were being altered [22]. Further- more, results indicated that the in vivo roll-over shape remained invariant across conditions apart from a decrease in the in vivo roll-over shape arc length (i.e., forefoot moment arm) with increasing number of cutouts [21]. Increasing forefoot flexibility resulted in increased peak prosthetic limb dorsiflexion during late stance [21], decreased prosthetic limb peak ankle flexion moment [21], and no effect on metabolic energy cost (milliliters of oxygen/kilogram/meter) when walking at a selfselected speed [22].

The roll-over shape model has also been used to characterize the effects of prosthesis alignment on gait. Hansen et al. showed that, when aligning different prostheses (of quite different AIPPs), prosthetists appear to converge on alignments for each of the prostheses that result in similar in vivo roll-over shapes [24]. The study clearly demonstrated that prosthetic devices with very different AIPPs could produce similar in vivo roll-over shapes. Further, the study suggests that the differences in gait behavior observed by clinicians when comparing feet are reasonably well explained by use of the roll-over model. Interestingly, however, the study did not go on to characterize in detail the changes to the mechanical properties as a result of alignment. This is important, as another study demonstrated that through changes in sagittal plane alignment, different AIPPs can be obtained with the same prosthetic foot [3]; alignment determines the points of contact of the foot during stance. Although many of the constituent materials may have isotropic properties, the assembled prosthesis will have anisotropic properties because of the nonsymmetric geometry of the subcomponents. This means that, in addition to translating and rotating the foot's geometry, alignment changes will also change the directions in which and the points at which the measured elastic and damping properties act relative to the residual anatomy.

\section{Simulation Studies That Have Used AIPP Roll-Over Models}

The roll-over shape model has recently been used in a computer simulation to investigate the effects of prosthesis alignment, prosthesis mass and mass distribution, and varying roll-over shape radius on the kinematics of amputee gait [41]. Gait kinematics were predicted using forward dynamic simulation of amputee gait, and the rollover shape was modeled as a circular arc. The study used an optimization approach to vary the roll-over shape radius and prosthesis alignment characteristics with the 
objective of minimizing both the total joint torque and joint power costs during gait. However, the roll-over model in this simulation was purely geometric and did not include stiffness and damping properties, factors that would influence joint torques and, hence, powers. Results indicated that lower total joint torque and joint power costs could be achieved by using a prosthetic roll-over shape radius that is equivalent to, or slightly larger than, the radius of the anatomical roll-over shape. Furthermore, the alignment, which minimized total joint torque and joint power costs, was found to be dependent on the rollover shape radius.

\section{Roll-Over Shape as Model for Representing AIPPs of Prosthetic Feet}

Overall, the roll-over shape model provides a clear and convincing way of explaining the influence of prosthetic foot geometry and alignment on gait. However, as commonly described in the literature (a single roll-over curve), it does not explicitly account for viscoelastic behavior, and hence, it is limited when used as a model with which to investigate ESAR or other dynamic effects. For example, two prostheses having the same in vivo roll-over shape, but different viscoelastic properties, may have different effects on impact loads at heel-strike or fatigue rates. Such effects might not be easily picked up during clinical gait observation by prosthetists when aligning prostheses, and this may explain the remarkable degree to which roll-over shape alone seems to determine alignment [24]. In fact, given that gait symmetry is a major factor in clinical prosthesis alignment, it is not surprising that geometry and kinematics dominate alignment rather than kinetics.

\section{Strengths and Limitations of AIPP Concept}

In this article, we have emphasized the concept of AIPPs (defined in the "Introduction"). Here we consider the merits and limitations of this concept.

The most important advantage is that of distinguishing between properties that describe the overall behavior of the prosthesis as directly experienced by the amputee (AIPPs) and the underlying design details (e.g., subcomponent geometries, material densities, and Young's moduli). The latter are not AIPPs; rather, they determine the AIPPs and, therefore, only indirectly affect amputee gait. This distinction must be made if we are to avoid an explosion in the number of properties used to describe prostheses. An illustrative analogy is that of a simple coil spring, the functional behavior of which can be succinctly described by just its uncompressed length and its stiffness, which are therefore analogous to the AIPPs of a prosthesis. Conversely, the properties of the spring material, the spring diameter, the diameter of the spring wire, and the number of turns making up the spring are the design details that determine its uncompressed length and stiffness. Many alternative designs could have the same uncompressed length and stiffness and they would all behave in the same way.

The sagittal plane behavior of a passive transtibial prosthesis during gait can be described by the following AIPPs:

- Two-dimensional roll-over shape.

- Alignment of the roll-over shape.

- Elastic properties.

- Damping properties.

- Mass properties.

The amputee independent roll-over shape captures the geometry of the prosthesis under a standard load. Together with the elastic properties, this describes the geometry under different loads. If the roll-over shape is defined in a socket-based reference frame, then it also captures alignment. The authors believe that, if described in sufficient detail (e.g., by extending the roll-over shape description to capture three-dimensional geometry), the AIPPs listed here provide a comprehensive description of the functional properties of a passive, transtibial prosthesis as experienced by the amputee. Other apparent properties, such as ranges of motion and ESAR behavior, are in fact determined by the AIPPs listed here and the individual amputee's characteristics. So these are not additional independent AIPPs.

Proper characterization of AIPPs is essential to the research stage of the design approach outlined in Figure 1. In order to systematically improve on existing designs, the properties of existing prostheses should be measured independent of the amputee (AIPPs) before they are subjected to in vivo performance testing. A better understanding of the correlations between AIPPs and their effects on amputee gait can then be developed through combined studies that measure both AIPPs and gait performance, with amputees grouped according to their motor capacities. In this way, desirable AIPPs could be established as inputs to the prosthesis design process (Figure 1). It is interesting to note that the vast majority of in vivo studies on amputee gait performance are constrained by the discrete set of AIPPs associated with commercially available feet. It is 
possible to envisage studies in which one AIPP is systematically varied at a time, without varying other AIPPs, which would make it easier to identify the effects of individual AIPPs. Such an approach may only be possible with experimental feet designed for this purpose, such as those used by Major [18], Ventura et al. [19-20], South et al. [44], and Klodd et al. [21-22]. Because of the importance of understanding the relationships between AIPPs and gait performance, we believe there needs to be a greater emphasis on controlled, systematic studies that properly characterize the AIPPs of the prostheses used and that, where possible, do not rely on commercial prosthetic components.

An AIPPs model could also be used as part of a gait simulation to explore the effects of AIPPs on gait, the advantages of which are that it allows for rapid and extensive design-test iterations that would not be possible with human subjects. Further, there are no constraints on the choice of AIPPs that can be tested within gait simulation. The predicted results from simulations may then be validated through carefully designed in vivo experimentation. The output of such studies would be desirable AIPPs, which could serve as the design criterion for the second stage of the approach described in Figure 1. One of the challenges of simulating amputee gait is that of representing different amputee populations with correspondingly different levels of motor capacity in order to determine the most appropriate AIPPs for each population. This could be achieved by constraining the simulated joint ranges of motion and joint moments to reflect a particular population's movement and motor capacity limitations.

If the AIPP approach to characterizing transtibial prostheses is to be of real value to the prosthetics industry and amputees, there needs to be agreement on the set of AIPPs and how they are defined. These definitions need to be sufficiently precise to ensure that AIPP measurements made by different researchers and manufacturers can be compared. Unfortunately, the prosthetics community has not yet achieved this level of agreement on the characterization of prostheses.

\section{CONCLUSIONS}

AIPPs can be represented as either lumped parameter or roll-over models. While using a one-degree-of-freedom lumped parameter model to represent a pylon shock- absorber element is clearly a reasonable approximation, it is argued that such models do not adequately represent the complex behavior of a prosthetic foot. With a small number of notable exceptions, most lumped parameter studies have chosen only to characterize the behavior of prosthetic feet at one or two locations. It is far from clear whether, for example, the stiffness at the heel and forefoot dominate the influence of prosthetic feet on gait, and hence, the benefit of simplifying the foot model to this extent is debatable. In contrast, the roll-over shape model provides a powerful way of representing the effects of loaded foot geometry and alignment over the length of the plantar surface. However, viscoelastic behavior and the response to shear loads are not yet included in the standard roll-over model. Furthermore, there are many inconsistencies in the AIPP definitions used, which can be either explicit or implicit (as a result of the measurement methods adopted), and this makes between-study comparisons very difficult. Therefore, based on this review, we have suggested a standardized method for stance phase AIPP characterization that combines the best features of lumped parameter and rollover models and may be employed in future in vivo and in silico research studies [57].

It should be noted that this review is limited to published articles that are available through online electronic databases, and we have not included any sources from industry-based designers and manufacturers of commercial prosthetic components, who do not disclose their design processes.

In summary, a variety of different approaches to AIPP characterization have been used to inform the interpretation of particular clinical studies. However, there appears to be no agreed-upon, standard approach to AIPP characterization. Further, methods and components used for characterization have sometimes been poorly explained, making replication of results difficult [5,13-15,30]. Both factors limit the extent to which the results of previous studies can be used to inform future designs. More importantly, until an agreed-upon approach to AIPPs is defined, it is difficult to conduct the systematic studies required to make rapid progress in the field. Most importantly, unless combined with in vivo gait analysis or gait simulation, AIPP characterization studies only provide part of the data necessary to inform improvements in prosthesis design through establishing associations between AIPP characteristics and measures of amputee performance [8]. Further, when undertaking combined in vivo and AIPP studies, it is 
important to control alignment in a systematic manner; it is an integral part of the AIPPs description of the prosthesis.

\section{ACKNOWLEDGMENTS}

\author{
Author Contributions: \\ Study concept and design: M. J. Major, L. P. J. Kenney, M. Twiste, \\ D. Howard. \\ Acquisition of data: M. J. Major. \\ Analysis and interpretation of data: M. J. Major, L. P. J. Kenney, \\ M. Twiste, D. Howard. \\ Drafting of manuscript: M. J. Major. \\ Critical revision of manuscript for important intellectual content: \\ M. J. Major, L. P. J. Kenney, M. Twiste, D. Howard. \\ Financial Disclosures: The authors have declared that no competing \\ interests exist. \\ Funding/Support: This material was based on work jointly funded \\ by the Whitaker International Fellows and Scholars Program of the \\ United States and the University of Salford, United Kingdom.
}

\section{REFERENCES}

1. Hafner BJ, Sanders JE, Czerniecki JM, Fergason J. Transtibial energy-storage-and-return prosthetic devices: a review of energy concepts and a proposed nomenclature. J Rehabil Res Dev. 2002;39(1):1-11. [PMID:11926321]

2. Hansen AH, Childress DS, Knox EH. Prosthetic foot rollover shapes with implications for alignment of trans-tibial prostheses. Prosthet Orthot Int. 2000;24(3):205-15.

[PMID:11195355] http://dx.doi.org/10.1080/03093640008726549

3. Hansen A. Effects of alignment on the roll-over shapes of prosthetic feet. Prosthet Orthot Int. 2008;32(4):390-402. [PMID:18985550] http://dx.doi.org/10.1080/03093640802366158

4. Selles RW, Bussmann JB, Wagenaar RC, Stam HJ. Effects of prosthetic mass and mass distribution on kinematics and energetics of prosthetic gait: a systematic review. Arch Phys Med Rehabil. 1999;80(12):1593-99. [PMID:10597812] http://dx.doi.org/10.1016/S0003-9993(99)90336-2

5. van Jaarsveld HW, Grootenboer HJ, de Vries J, Koopman HF. Stiffness and hysteresis properties of some prosthetic feet. Prosthet Orthot Int. 1990;14(3):117-24. [PMID:2095529]

6. Geil MD. Energy loss and stiffness properties of dynamic elastic response prosthetic feet. J Prosthet Orthot. 2001; 13(3):70-73. http://dx.doi.org/10.1097/00008526-200109000-00011

7. Postema K, Hermens HJ, de Vries J, Koopman HF, Eisma WH. Energy storage and release of prosthetic feet. Part 1:
Biomechanical analysis related to user benefits. Prosthet Orthot Int. 1997;21(1):17-27. [PMID:9141122]

8. Hafner BJ, Sanders JE, Czerniecki J, Fergason J. Energy storage and return prostheses: does patient perception correlate with biomechanical analysis? Clin Biomech (Bristol, Avon). 2002;17(5):325-44. [PMID:12084537] http://dx.doi.org/10.1016/S0268-0033(02)00020-7

9. Klute GK, Kallfelz CF, Czerniecki JM. Mechanical properties of prosthetic limbs: adapting to the patient. J Rehabil Res Dev. 2001;38(3):299-307. [PMID:11440261]

10. Hofstad C, Linde H, Limbeek J, Postema K. Prescription of prosthetic ankle-foot mechanisms after lower limb amputation. Cochrane Database Syst Rev. 2004;(1):CD003978. [PMID:14974050]

11. van der Linde H, Hofstad CJ, Geurts AC, Postema K, Geertzen JH, van Limbeek J. A systematic literature review of the effect of different prosthetic components on human functioning with a lower-limb prosthesis. J Rehabil Res Dev. 2004;41(4):555-70. [PMID:15558384] http://dx.doi.org/10.1682/JRRD.2003.06.0102

12. Twiste M, Rithalia S. Transverse rotation and longitudinal translation during prosthetic gait-a literature review. J Rehabil Res Dev. 2003;40(1):9-18. [PMID:15150716] http://dx.doi.org/10.1682/JRRD.2003.01.0009

13. Lehmann JF, Price R, Boswell-Bessette S, Dralle A, Questad K. Comprehensive analysis of dynamic elastic response feet: Seattle Ankle/Lite Foot versus SACH foot. Arch Phys Med Rehabil. 1993;74(8):853-61. [PMID:8347071] http://dx.doi.org/10.1016/0003-9993(93)90013-Z

14. Lehmann JF, Price R, Boswell-Bessette S, Dralle A, Questad $\mathrm{K}$, deLateur BJ. Comprehensive analysis of energy storing prosthetic feet: Flex Foot and Seattle Foot versus standard SACH foot. Arch Phys Med Rehabil. 1993; 74(11):1225-31. [PMID:8239969]

15. Miller LA, Childress DS. Analysis of a vertical compliance prosthetic foot. J Rehabil Res Dev. 1997;34(1):52-57. [PMID:9021625]

16. Hansen AH, Meier MR, Sessoms PH, Childress DS. The effects of prosthetic foot roll-over shape arc length on the gait of trans-tibial prosthesis users. Prosthet Orthot Int. 2006;30(3):286-99. [PMID:17162519] http://dx.doi.org/10.1080/03093640600816982

17. vd Water GJ, de Vries J, Mulder MA. Comparison of the lightweight Camp Normal Activity Foot with other prosthetic feet in trans-tibial amputees: a pilot study. Prosthet Orthot Int. 1998;22(2):107-14. [PMID:9747994]

18. Major MJ. The influence of the mechanical properties of trans-tibial prostheses on amputee performance [dissertation]. [Salford, UK]: University of Salford; 2010.

19. Ventura JD, Klute GK, Neptune RR. The effect of prosthetic ankle energy storage and return properties on muscle activity in below-knee amputee walking. Gait Posture. 
2011;33(2):220-26. [PMID:21145747]

http://dx.doi.org/10.1016/j.gaitpost.2010.11.009

20. Ventura JD, Klute GK, Neptune RR. The effects of prosthetic ankle dorsiflexion and energy return on below-knee amputee leg loading. Clin Biomech (Bristol, Avon). 2011; 26(3):298-303. [PMID:21093131] http://dx.doi.org/10.1016/j.clinbiomech.2010.10.003

21. Klodd E, Hansen A, Fatone S, Edwards M. Effects of prosthetic foot forefoot flexibility on gait of unilateral transtibial prosthesis users. J Rehabil Res Dev. 2010;47(9):899-910. [PMID:21174254] http://dx.doi.org/10.1682/JRRD.2009.10.0166

22. Klodd E, Hansen A, Fatone S, Edwards M. Effects of prosthetic foot forefoot flexibility on oxygen cost and subjective preference rankings of unilateral transtibial prosthesis users. J Rehabil Res Dev. 2010;47(6):543-52. [PMID:20848367] http://dx.doi.org/10.1682/JRRD.2010.01.0003

23. Lee WC, Zhang M, Chan PP, Boone DA. Gait analysis of low-cost flexible-shank transtibial prostheses. IEEE Trans Neural Syst Rehabil Eng. 2006;14(3):370-77. [PMID:17009497] http://dx.doi.org/10.1109/TNSRE.2006.881540

24. Hansen AH, Meier MR, Sam M, Childress DS, Edwards ML. Alignment of trans-tibial prostheses based on roll-over shape principles. Prosthet Orthot Int. 2003;27(2):89-99. [PMID:14571938] http://dx.doi.org/10.1080/03093640308726664

25. Geil MD. An iterative method for viscoelastic modeling of prosthetic feet. J Biomech. 2002;35(10):1405-10. [PMID:12231286] http://dx.doi.org/10.1016/S0021-9290(02)00169-0

26. Kabra SG, Narayanan R. Equipment and methods for laboratory testing of ankle-foot prostheses as exemplified by the Jaipur foot. J Rehabil Res Dev. 1991;28(3):23-34. [PMID:1880747] http://dx.doi.org/10.1682/JRRD.1991.07.0023

27. Berge JS, Klute GK, Czerniecki JM. Mechanical properties of shock-absorbing pylons used in transtibial prostheses. J Biomech Eng. 2004;126(1):120-22. [PMID:15171138] http://dx.doi.org/10.1115/1.1645865

28. Skinner HB, Abrahamson MA, Hung RK, Wilson LA, Effeney DJ. Static load response of the heels of SACH feet. Orthopedics. 1985;8(2):225-28. [PMID:4094971]

29. Klute GK, Berge JS, Segal AD. Heel-region properties of prosthetic feet and shoes. J Rehabil Res Dev. 2004;41(4): 535-46. [PMID:15558382] http://dx.doi.org/10.1682/JRRD.2003.02.0025

30. Saunders MM, Schwentker EP, Kay DB, Bennett G, Jacobs CR, Verstraete MC, Njus GO. Finite element analysis as a tool for parametric prosthetic foot design and evaluation. Technique development in the solid ankle cushioned heel
(SACH) foot. Comput Methods Biomech Biomed Engin. 2003;6(1):75-87. [PMID:12623440]

http://dx.doi.org/10.1080/1025584021000048974

31. Flick KC, Orendurff MS, Berge JS, Segal AD, Klute GK. Comparison of human turning gait with the mechanical performance of lower limb prosthetic transverse rotation adapters. Prosthet Orthot Int. 2005;29(1):73-81.

[PMID:16180379]

http://dx.doi.org/10.1080/03093640500088120

32. Sam M, Hansen AH, Childress DS. Mechanical characterization of prosthetic feet using a prosthetic foot loading apparatus. Proceedings of the 22nd Annual International Conference of the IEEE Engineering in Medicine and Biology Society; 2000 Jul 23-28; Evanston, IL. p. 1968-71.

33. Curtze C, Hof AL, van Keeken HG, Halbertsma JP, Postema K, Otten B. Comparative roll-over analysis of prosthetic feet. J Biomech. 2009;42(11):1746-53.

[PMID:19446814]

http://dx.doi.org/10.1016/j.jbiomech.2009.04.009

34. Sam M, Hansen AH, Childress DS. Characterisation of prosthetic feet used in low-income countries. Prosthet Orthot Int. 2004;28(2):132-40. [PMID:15382807] http://dx.doi.org/10.1080/03093640408726698

35. Twiste M, Rithalia SV, Kenney L. A cam-displacement transducer device for measuring small two-degree of freedom inter-component motion in a prosthesis. Med Eng Phys. 2004;26(4):335-40. [PMID:15121059] http://dx.doi.org/10.1016/j.medengphy.2004.01.002

36. Coleman KL, Boone DA, Smith DG, Czerniecki JM. Effect of trans-tibial prosthesis pylon flexibility on ground reaction forces during gait. Prosthet Orthot Int. 2001;25(3): 195-201. [PMID:11860093] http://dx.doi.org/10.1080/03093640108726602

37. Rogers JP, Strike SC, Wallace ES. The effect of prosthetic torsional stiffness on the golf swing kinematics of a left and a right-sided trans-tibial amputee. Prosthet Orthot Int. 2004;28(2):121-31. [PMID:15382806] http://dx.doi.org/10.1080/03093640408726697

38. Klute GK, Berge JS. Modelling the effect of prosthetic feet and shoes on the heel-ground contact force in amputee gait. Proc Inst Mech Eng H. 2004;218(3):173-82. [PMID:15239568] http://dx.doi.org/10.1243/095441104323118897

39. Zmitrewicz RJ, Neptune RR, Sasaki K. Mechanical energetic contributions from individual muscles and elastic prosthetic feet during symmetric unilateral transtibial amputee walking: a theoretical study. J Biomech. 2007; 40(8):1824-31. [PMID:17045595] http://dx.doi.org/10.1016/j.jbiomech.2006.07.009

40. Knox EH. The role of prosthetic feet in walking [dissertation]. [Evanston, IL]: Northwestern University; 1996. 
41. Srinivasan S, Westervelt ER, Hansen AH. A low-dimensional sagittal-plane forward-dynamic model for asymmetric gait and its application to study the gait of transtibial prosthesis users. J Biomech Eng. 2009;131(3):031003.

[PMID:19154062]

http://dx.doi.org/10.1115/1.3002757

42. Zeller S. Stiffness properties of prosthetic feet under crossslope conditions [Master's thesis]. [Atlanta, GA]: Georgia Institute of Technology; 2007.

43. Wilson JR, Asfour S, Abdelrahman KZ, Gailey R. A new methodology to measure the running biomechanics of amputees. Prosthet Orthot Int. 2009;33(3):218-29.

[PMID:19658012]

http://dx.doi.org/10.1080/03093640903107998

44. South BJ, Fey NP, Bosker G, Neptune RR. Manufacture of energy storage and return prosthetic feet using selective laser sintering. J Biomech Eng. 2010;132(1):015001. [PMID:20524754] http://dx.doi.org/10.1115/1.4000166

45. Fung YC, Tong P. Classical and computational solid mechanics. 1st ed. London (UK): World Scientific Publishing Co. Pte. Ltd.; 2001.

46. Berge JS, Czerniecki JM, Klute GK. Efficacy of shockabsorbing versus rigid pylons for impact reduction in transtibial amputees based on laboratory, field, and outcome metrics. J Rehabil Res Dev. 2005;42(6):795-808.

[PMID:16680617]

http://dx.doi.org/10.1682/JRRD.2005.02.0034

47. Gard SA, Konz RJ. The effect of a shock-absorbing pylon on the gait of persons with unilateral transtibial amputation. J Rehabil Res Dev. 2003;40(2):109-24.

[PMID:15077637]

http://dx.doi.org/10.1682/JRRD.2003.03.0109

48. Jones SF, Twigg PC, Scally AJ, Buckley JG. The mechanics of landing when stepping down in unilateral lower-limb amputees. Clin Biomech (Bristol, Avon). 2006;21(2):184-93. [PMID:16274904]

http://dx.doi.org/10.1016/j.clinbiomech.2005.09.015

49. Adderson JA, Parker KE, Macleod DA, Kirby RL, McPhail C. Effect of a shock-absorbing pylon on transmission of heel strike forces during the gait of people with unilateral trans-tibial amputations: a pilot study. Prosthet Orthot Int. 2007;31(4):384-93. [PMID:18050009]

http://dx.doi.org/10.1080/03093640701254018

50. Klute GK, Berge JS, Orendurff MS, Williams RM, Czerniecki JM. Prosthetic intervention effects on activity of lower-extremity amputees. Arch Phys Med Rehabil. 2006; 87(5):717-22. [PMID:16635636] http://dx.doi.org/10.1016/j.apmr.2006.02.007

51. Buckley JG, Jones SF, Birch KM. Oxygen consumption during ambulation: comparison of using a prosthesis fitted with and without a tele-torsion device. Arch Phys Med
Rehabil. 2002;83(4):576-80. [PMID:11932866]

http://dx.doi.org/10.1053/apmr.2002.30624

52. Segal AD, Orendurff MS, Czerniecki JM, Shofer JB, Klute GK. Local dynamic stability of amputees wearing a torsion adapter compared to a rigid adapter during straight-line and turning gait. J Biomech. 2010;43(14):2798-803.

[PMID:20719315]

http://dx.doi.org/10.1016/j.jbiomech.2010.05.038

53. Segal AD, Orendurff MS, Czerniecki JM, Shofer JB, Klute GK. Transtibial amputee joint rotation moments during straight-line walking and a common turning task with and without a torsion adapter. J Rehabil Res Dev. 2009;46(3): 375-83. [PMID:19675989] http://dx.doi.org/10.1682/JRRD.2008.06.0070

54. Su PF, Gard SA, Lipschutz RD, Kuiken TA. The effects of increased prosthetic ankle motions on the gait of persons with bilateral transtibial amputations. Am J Phys Med Rehabil. 2010;89(1):34-47. [PMID:20026945] http://dx.doi.org/10.1097/PHM.0b013e3181c55ad4

55. Prince F, Winter DA, Sjonnesen G, Wheeldon RK. A new technique for the calculation of the energy stored, dissipated, and recovered in different ankle-foot prostheses. IEEE Trans Rehabil Eng. 1994;2(4):247-55. http://dx.doi.org/10.1109/86.340873

56. Geil MD, Parnianpour M, Quesada P, Berme N, Simon S. Comparison of methods for the calculation of energy storage and return in a dynamic elastic response prosthesis. J Biomech. 2000;33(12):1745-50. [PMID:11006404] http://dx.doi.org/10.1016/S0021-9290(00)00102-0

57. Major MJ, Twiste M, Kenney LP, Howard D. Amputee Independent Prosthesis Properties - a new model for description and measurement. J Biomech. 2011;44(14):2572-75.

[PMID:21831379]

http://dx.doi.org/10.1016/j.jbiomech.2011.07.016

Submitted for publication June 10, 2011. Accepted in revised form November 23, 2011.

This article and any supplementary material should be cited as follows:

Major MJ, Kenney LP, Twiste M, Howard D. Stance phase mechanical characterization of transtibial prostheses distal to the socket: A review. J Rehabil Res Dev. 2012;49(6): 815-30.

http://dx.doi.org/10.1682/JRRD.2011.06.0108

ResearcherID: Matthew J. Major, PhD: E-7372-2012

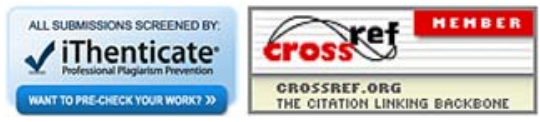


